\title{
Rapid Detection of Sialidase Activity for the Diagnosis of Bacterial Vaginosis
}

\author{
Jari Intra ${ }^{1 *}$, Cecilia Sarto ${ }^{1}$, Natalia Tiberti ${ }^{2}$, Claudia Siracusa ${ }^{1}$, \\ Cinzia Savarino", Chiara Fania ${ }^{3}$ and Paolo Brambilla ${ }^{1}$ \\ ${ }^{1}$ Department of Laboratory Medicine, University of Milano-Bicocca, Desio Hospital, \\ via Mazzini 1, 20833, Desio (MB), Italy \\ ${ }^{2}$ Centre for Tropical Diseases, IRCCS Sacro Cuore Don Calabria Hospital, Negrar, \\ Verona, Italy \\ ${ }^{3}$ Dipartimento di Medicina e Chirurgia, Università degli Studi Milano-Bicocca, Milano, Italy \\ *Corresponding author
}

\begin{tabular}{|l|}
\hline K e y w o r d s \\
Bacterial vaginosis, \\
$\begin{array}{l}\text { Nugent's score, OSOM } \\
\text { BVBlue test, Vaginal } \\
\text { microbial cultures, } \\
\text { Bacterial sialidase }\end{array}$ \\
\hline Article Info \\
\hline $\begin{array}{l}\text { Accepted: } \\
\text { 22 July 2018 } \\
\text { Available Online: } \\
\text { 10 August } 2018\end{array}$ \\
\hline
\end{tabular}

\section{Introduction}

Bacterial vaginosis (BV) is the most common cause of vaginal discharge. This pathology is characterized by a shift in the flora from the normally predominant Lactobacillus (Spiegel, 1991; Smayevsky et al., 2001) to one
Bacterial vaginosis (BV) is one of the most frequent causes of vaginal discharge in women during reproductive age worldwide. This disease is characterized by the replacement of the normal vaginal flora with an overgrowth of anaerobic bacteria most of them producing sialidase enzyme. BV is associated with an increased risk of adverse outcomes in pregnancy and susceptibility to several sexually transmitted diseases. In the present study, we evaluated the detection of sialidase activity by OSOM BVBlue test in association with routine microbial cultures and Nugent's score, considered as the gold standard, for the diagnosis of bacterial vaginosis. Three vaginal swabs were collected from 352 women older than 12 years in age. A swab collected into Amies transport medium was employed for standard microbial cultures, a FLOQSwab for Gram stain, and a second FLOQSwab for the BVBlue test. According to Nugent's score, BV frequency was $16.5 \%$ (58 samples). The sensitivity of microbial culture and BVBlue test, when compared with Nugent's score, was $69.8 \%$ and $39.6 \%$, respectively. However, BVBlue test detected five cases with no bacterial growth in culture, whereas 14 samples with bacterial cultures positive for Gardnerella vaginalis showed a BVBlue test negative. The combination of microbial culture and BVBlue test increased the sensitivity to $75 \%$ compared with Nugent's score. In conclusion, BVBlue test alone appears not to be an efficient screening test, but, when associated with microbial cultures, can improve the diagnosis of BV. 
associated with adverse health outcomes, such as preterm delivery (Hillier et al., 1995; Howe et al., 1999), pelvic inflammatory disease (Spiegel, 1991; Sweet, 1995), and endometritis (von Nicolai et al., 1984; Haggerty et al., 2004). Moreover, a strong association with increased susceptibility to infections due to Herpes simplex virus 2, Chlamydia trachomatis, Trichomonas vaginalis, Neisseria gonorrheae, and human immunodeficiency virus (HIV) has been reported (Bhalla et al., 2007; Bagnall and Rizzolo, 2017; Lokken et al., 2017). Different investigations are performed to diagnose $\mathrm{BV}$, including Gram staining and cultures for Gardnerella vaginalis as well as for other aerobic/facultative anaerobic organisms involved in vaginal infections. Gram smear examination based on Nugent's system and Amsel's criteria is traditionally used in the diagnosis of bacterial vaginosis (Spiegel et al., 1983; Nugent et al., 1991), but needs microbiology expertise. Moreover, cultures for anaerobic organisms are not routinely carried out. Anaerobic bacteria involved in BV can be identified using molecular techniques, which have been recently introduced in microbiology laboratories (Ling et al., 2010; Kusters et al., 2015; Rumyantseva et al., 2015; Gaydos et al., 2017; Virtanen et al., 2017). These methods are however expensive and require trained and expert personnel, limiting their application in routine diagnosis.

In the last fifteen years, the detection of the activity of microbial enzymes, including sialidases, in vaginal fluid has been demonstrated to be useful for the rapid diagnosis of BV (Wiggins et al., 2000, 2001).

These enzymes are present in several bacteria, viruses, mycoplasma, fungi, and protozoa (Von Nicolai et al., 1984; Taylor, 1996), and have been reported to play a role in nutrition, cellular interactions, and immune response. Additionally, they have been shown to improve the adhesion, invasion and destruction of mucosal tissues by bacteria (Briselden et al., 1992; Cauci et al., 1998; Wiggins et al., 2000; Smayevsky et al., 2001). Interestingly, anaerobic gram-negative bacteria involved in bacterial vaginosis, such as Bacteroides, Gardnerella, Atopobium, Mobiluncus, and Prevotella spp., are known to secrete sialidases (Moncla, et al., 1990; Briselden et al., 1992; Cauci et al., 1998).

To improve BV diagnosis, a rapid chromogenic method for the detection of sialidase activity in vaginal fluids, the OSOM BVBlue system (Gryphus Diagnostics, Birmingham, AL, USA), has recently been developed. The aim of our study was to evaluate if the OSOM BVBlue test in association with routine microbial culture and Gram staining (Nugent's score) can improve the diagnosis of BV by detecting the sialidase activity produced by anaerobic bacteria, which otherwise could not be routinely detect in vaginal discharge.

\section{Materials and Methods}

\section{Study population}

A total of 352 consecutive non-pregnant and unselected women in the reproductive age (12 to 50 years old), admitted to the Hospital of Desio (Lombardy, Italy) from September 2016 to August 2017 with an abnormal vaginal discharge recorded by the clinicians during speculum examination, were enrolled in this study. Written informed consent was obtained from all women recruited. Three samples were taken from the vaginal wall using swabs: one swab was collected into liquid Amies Transport Medium (ESwab) (Copan Flock Technologies S.r.1., Brescia, Italy), while two sterile FLOQSwabs (Copan Flock Technologies S.r.l., Brescia, Italy) were used to prepare Gram stained smears and to perform the OSOM BVBlue test. 


\section{Laboratory assessments}

The swab collected into liquid Amies Transport Medium was inoculated on selective agar plates: Rogosa Agar (Oxoid, Cheshire, $\mathrm{UK}$ ), for the isolation and count of Lactobacilli spp.; Columbia agar with $5 \%$ sheep blood (COS, bioMérieux, Marcy l'Etoile, France), for the growth and isolation of fastidious and non-fastidious microorganisms; Candida Agar (CAN2, bioMérieux, Marcy l'Etoile, France), for the isolation of fungi and the direct identification of Candida albicans; TRIS agar plate (composed of modified Thayer Martin Agar, Chocolate agar enriched and Gardnerella selective agar) for the isolation of Gardnerella vaginalis and Neisseria spp. (Thermo Fisher Scientific, Rodano, Italy); OSOM Trichomonas rapid test for the detection of Trichomonas vaginalis (Seisuki Diagnostics Allington Maidstone, Kent, UK). COS and TRIS agar plates were incubated for 24-48 hours at $36^{\circ} \mathrm{C} \pm 1{ }^{\circ} \mathrm{C}$ in $5 \% \mathrm{CO}_{2}$; Rogosa agar plates were incubated under anaerobic conditions for $24-48$ hours at $36^{\circ} \mathrm{C} \pm 1{ }^{\circ} \mathrm{C}$; CAN2 agar plates were incubated for 48 hours at $36^{\circ} \mathrm{C} \pm 1{ }^{\circ} \mathrm{C}$.

Gram staining was carried out on two smears using one of the FLOQSwabs to assess normal vaginal flora, or the presence of fungi, polymorphous-nuclear cells, and clue cells. Two different trained laboratory technicians evaluated the smears in a blinded manner to minimize potential biases. Nugent's criteria, considered the gold standard for $\mathrm{BV}$ diagnosis, were applied and a score from zero to ten was assigned based on the presence of three bacterial morphotypes: (1) long Grampositive rods (Lactobacillus spp.), (2) small Gram-negative, Gram-variable rods and cocci (Gardnerella, Prevotella, Porphyromonas, and Bacteroides spp.), (3) curved Gramnegative rods (Mobiluncus spp.). A score < 3 was considered as normal bacterial vaginal flora thus negative for bacterial vaginosis, a score between 4 and 6 was indicative of an altered vaginal flora not consistent with bacterial vaginosis, and a score > 6 was indicative of bacterial vaginosis (Nugent et al., 1991).

The third swab was used to perform OSOM BVBlue test (Seisuki Diagnostics Allington Maidstone, Kent, UK) according to manufacturer's instruction. A blue or green color was considered as positive result, indicating a high level of sialidase activity, while a yellow color was considered negative, indicating a normal level of sialidase activity. The minimum detection limit of sialidase activity was $7.46 \mathrm{U}$ (equivalent to $0.25 \mu \mathrm{g}$ ).

\section{MALDI-TOF MS identification}

Bacterial colonies grown on each agar plate were scraped using a $1-\mu 1$ disposable plastic loop, picked in duplicate and directly transferred without any additional step on the same VITEK $^{\circledR} \quad$ MS-DS target slide (bioMérieux). Each sample was covered with $1 \mu \mathrm{l}$ of saturated $\alpha$-Cyano-4-hydroxycinnamic acid (CHCA) in $50 \%$ acetonitrile and $2.5 \%$ trifluoracetic acid matrix solution $\left(\mathrm{VITEK}^{\circledR}\right.$ MS-CHCA, bioMérieux), and processed using MALDI-TOF VITEK ${ }^{\circledR}$ MS RUO system (bioMérieux). The results were analyzed using the Saramis ${ }^{\mathrm{TM}}$ database (Spectral ARchive and Microbial Identification System) (Version 4.10) (AnagnosTec) and Shimadzu Biotech Launchpad $^{\circledR}$ software. All mass fingerprints were compared to the superspectra and individual spectra of the database, and the results were expressed as percentage of similarity. Data analysis was performed following using manufacturer's instructions, thus identifications with similarity between 75 and $99.9 \%$ similarity were considered valid at the species level, while spectra with similarity lower than $75 \%$ were considered nonidentified. These confidence levels are based 
on the goodness of fit to weighted consensus reference spectra for a given taxon. Each target slide was calibrated and validated with the Escherichia coli ATCC $^{\circledR} 8739$ strain as control.

\section{Statistical analysis}

Statistical tests, sensitivity, and specificity were calculated using MedCalc for Windows, version 15.0 (MedCalc Software, Ostend, Belgium) (Stephan et al., 2003).

\section{Results and Discussion}

In 238 out of 352 samples, different microorganisms were isolated. The most frequent causative pathogens detected after growth on culture media and identified using MALDI-TOF MS were Candida albicans (45.4 \%), Gardnerella vaginalis (28.1\%), Candida glabrata (8.7 \%), and Enterococcus faecalis $(3.4 \%)$ (Table 1). In nine samples, more than one pathogen was detected (Table 1). Initially, the microscopic examination assigned a Nugent's score $<4$ to 256 samples $(72.7 \%)$, and $>6$, thus consistent with the presence of bacterial vaginosis, to 58 subjects $(16.5 \%)$. An altered vaginal flora (Nugent's score: 4-6) was observed in 38 cases (10.8\%). Clue cells, i.e. epithelial cells coated with bacteria, were observed in $100 \%$ of samples having a Nugent's score $>6$. Trichomonas vaginalis was found in three specimens presenting a Nugent's score $<4$.

Endocervical swabs of all the 352 subjects enrolled in this study were used to detect the infection due to Chlamydia trachomatis and Mycoplasma spp. Sixty-one samples were positive for Mycoplasma spp. while only one sample for Chlamydia trachomatis. The presence of these bacteria did not exclude the possibility of co-infections with other pathogens, thus those vaginal swabs were used for subsequent analyses, including the BVBlue test, despite Nugent's score was lower than 4 in all of these samples.

Gardnerella vaginalis was identified in 32 out of the 58 samples with Nugent's score $>6$ (55.2\%), 14 of which showed a negative BVBlue test. Moreover, 26 samples (44.8\%) had negative microbial cultures, but five of these showed a positive BVBlue test (Table 2). Among these, after microscopic examination, four revealed the presence of small Gram-negative bacteria similar to Prevotella/Bactereoides spp., and one sample exhibited rod-shaped curved Gram variable bacteria similar to Mobiluncus spp. Last, in only one sample presenting a Nugent's score $>$ 6 and a co-infection of Gardnerella vaginalis and Candida albicans, BVBlue test resulted positive.

Among the 38 samples with a Nugent's score between four and six, Gardnerella vaginalis was identified in 35 samples $(92.1 \%)$, and BVBlue test was positive in 15 (39.5\%), and negative in $23(60.5 \%)$. At last, BVBlue test resulted negative in all the 256 samples with a Nugent's score $<4$.

Collectively, considering the samples with a Nugent's score $>4$, BVBlue test was positive in $38(39.6 \%)$, and negative in $58(60.4 \%)$ of them (Table 3 ). The correlation of the results obtained with the three diagnostic tests (Gram staining with microscopic examination, microbial growth, and BVBlue test) showed that, among the 96 cases with a Nugent's score $>4,33$ (15 samples with Nugent's score 4-6 and 18 with Nugent's score $>6$ ) were detected as positive by all tests. In 24 cases (three samples with Nugent's score 4-6 and 21 with Nugent's score $>6$ ), microbial cultures and BVBlue test were instead negative. When compared with microscopic examination (Nugent's score), BVBlue test and microbial cultures showed a specificity of $100 \%$ and a sensitivity of $39.6 \%$ and $69.8 \%$, respectively. 
The combination of microbial growth on selective agar plates and BVBlue test increased the sensitivity to $75 \%$ (Table 3 ).

In the present study, we have evaluated the utility of bacterial sialidase activity detection using the combination of OSOM BVBlue test and microbial cultures for BV diagnosis, and considering the Nugent's score as the gold standard. Our results showed a low sensitivity of BVBlue test $(39.6 \%$ ) for the detection of Nugent's score higher than four, indicative of altered vaginal flora and suggestive of bacterial vaginosis. Similar results were also obtained in a recent study of Madhivanan et al., (2014) pointing out that BVBlue had 38.1 $\%$ sensitivity when performed alone. In contrast, previous works reported a sensitivity of BVBlue test ranging from $88 \%$ to $100 \%$ (Myziuk et al., 2003; Bradshaw et al., 2005; Shujatullah et al., 2010; Kampan et al., 2011; Khatoon et al., 2013). The good quality of our analysis was ensured at different levels. Firstly, in our study, trained and experienced personnel collected each sample, and the
BVBlue test was carried out within a short time (within $2 \mathrm{~h}$ ), ensuring the quality of the specimens for the analysis. The sterile cotton swabs provided within the BVBlue kit (validated for use) are packaged in paper containers that can be used in a physician's office but are not assembled into cup-sealed sterile tubes necessary for a safety transport to the laboratory. We thus used short flocked Nylon® fiber swabs for sample collection to overcome this drawback. However, these swabs cannot be considered as a possible cause of false-negative BVBlue test results since we did not observe any differences in the results obtained with the two type of swabs soaked in G. vaginalis positive and negative microbial cultures (data not shown). Secondly, in the evaluation of Nugent's score, experienced microbiologists, blind to the other results, examined the Gram-stained smears reducing possible systematic human errors. Finally, the instructions provided with the kit were strictly followed and detailed guidelines were provided to the patients for an appropriate preparation before sampling.

\section{Table.1 Microorganisms associated with vaginal infection identified by MALDI-TOF MS}

\begin{tabular}{|l|c|c|} 
& \multicolumn{2}{c|}{ Total $(n=238,100 \%)$} \\
\hline Candida albicans & $\boldsymbol{n}$ & $\%$ \\
\hline Gardnerella vaginalis & 108 & 45.4 \\
\hline Candida glabrata & 66 & 27.7 \\
\hline Enterococcus faecalis & 21 & 8.8 \\
\hline Staphilococcus aureus & 8 & 3.5 \\
\hline Candida krusei & 5 & 2.1 \\
\hline Prevotella disiens & 4 & 1.8 \\
\hline Trichomonas vaginalis & 4 & 1.8 \\
\hline Enterococcus faecium & 3 & 1.4 \\
\hline Neisseria gonorrhoeae & 2 & 0.8 \\
\hline Candida parapsilosis & 2 & 0.8 \\
\hline Mobiluncus spp. & 2 & 0.8 \\
\hline Morganella morganii & 1 & 0.4 \\
\hline Candida tropicalis & 1 & 0.4 \\
\hline Mixed pathogens: & 1 & 0.4 \\
\hline Candida albicans + Enterococcus faecalis & & \\
\hline Candida albicans + Gardnerella vaginalis & & \\
\hline
\end{tabular}


Table.2 Cases of Bacterial vaginosis detected by microbial culture and BVBlue test

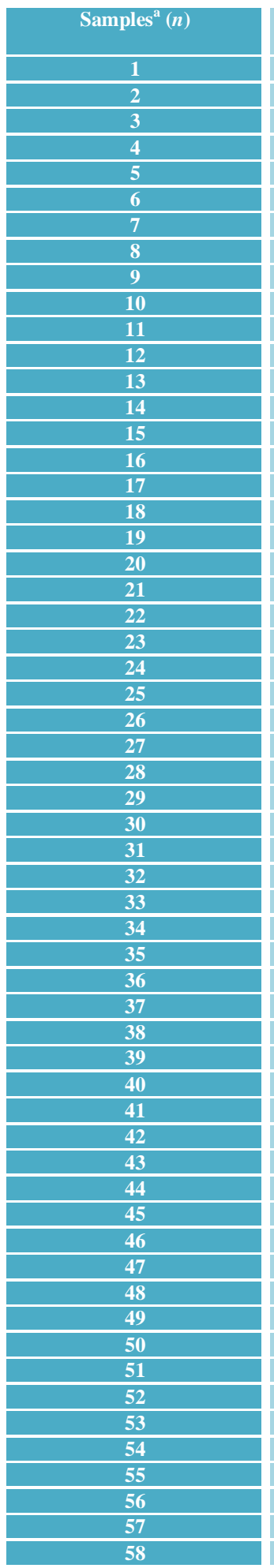

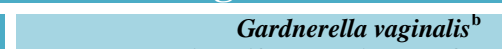

identifed by MALDI-TOF MS

Negative

Negative

Negative

Negative

Negative

Negative

Negative

Negative

Negative

Negative

Positive

Negative

Negative

Negative

Positive

Positive

Positive

Positive

Positive

Negative

Negative

Positive

Positive

Positive

Negative

Negative

Positive

Negative

Positive

Positive

Positive

Positive

Positive

Positive

Negative

Positive

Positive

Positive

Negative

Positive

Positive

Positive

Positive

Positive

Negative

Positive

Positive

Negative

Negative

Positive

Positive

Negative

Positive

Negative

Positive

Positive

Negative

Positive
OSOM BVBlue test

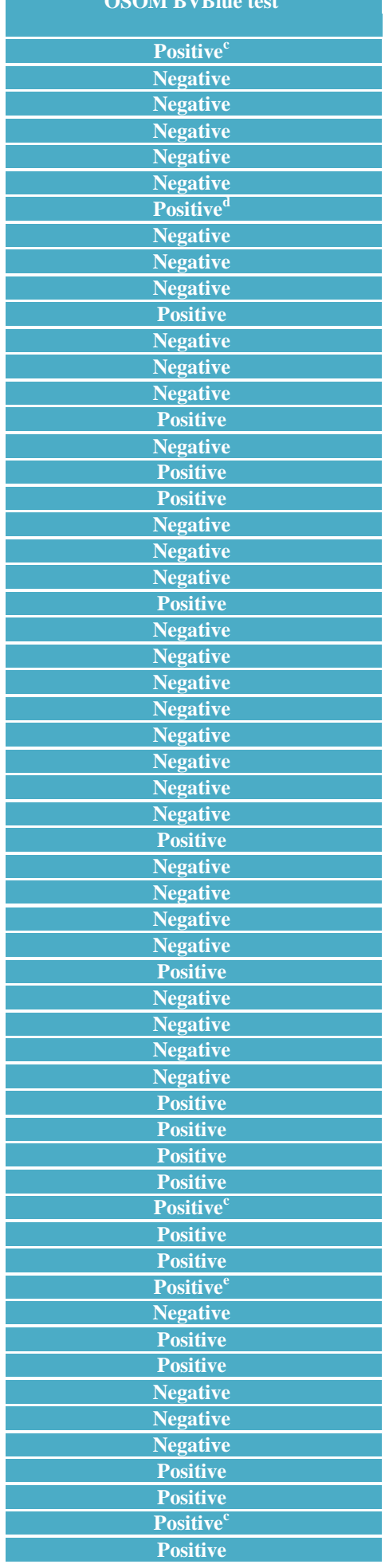

'Subjects with Nugent's score greater than six (See Methods).

${ }^{b}$ Microbial culture performed on selective agar plate for Gardnerella vaginalis.

${ }^{c}$ Microscopic examination: small Gram negative bacteria like to Prevotella/Bacteroides spp.

${ }^{\mathrm{d}}$ Microscopic examination: rod-shaped curved Gram variable bacteria similar to Mobiluncus spp.

eMicroscopic examination: rod-shaped Gram variable bacteria like to Gardnerella vaginalis. 
Int.J.Curr.Microbiol.App.Sci (2018) 7(8): 3898-3908

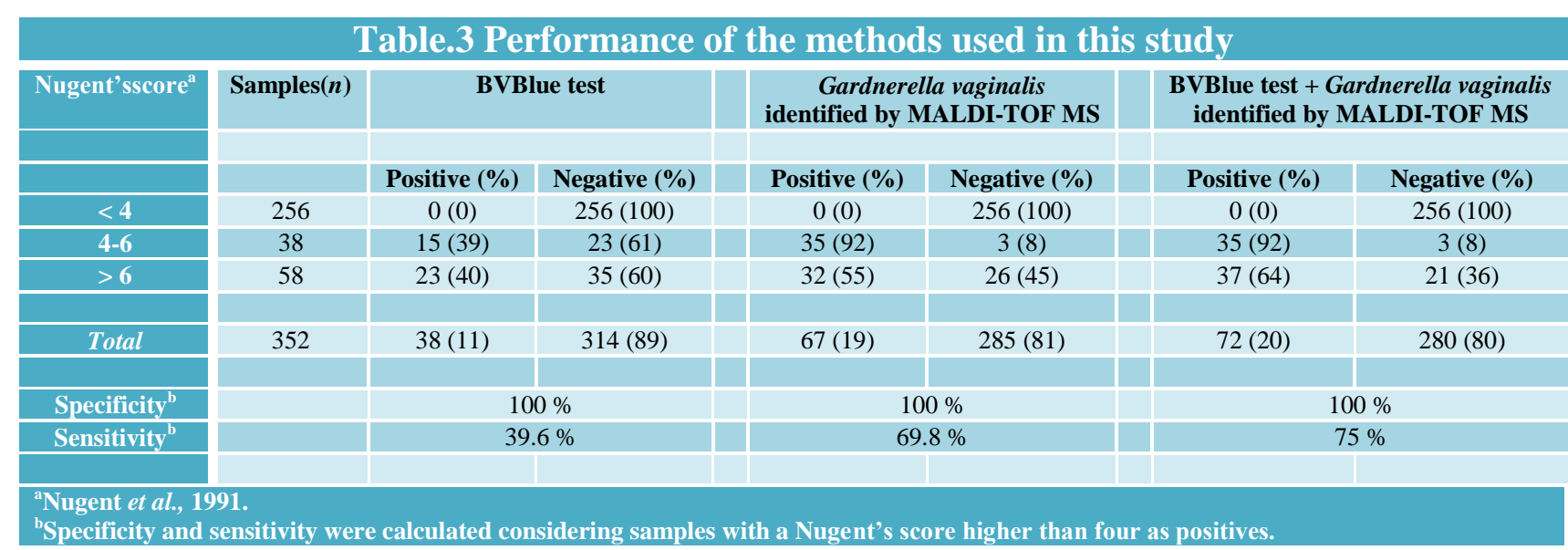

Fig.1 Flow chart proposed for a rapid diagnosis of bacterial vaginosis

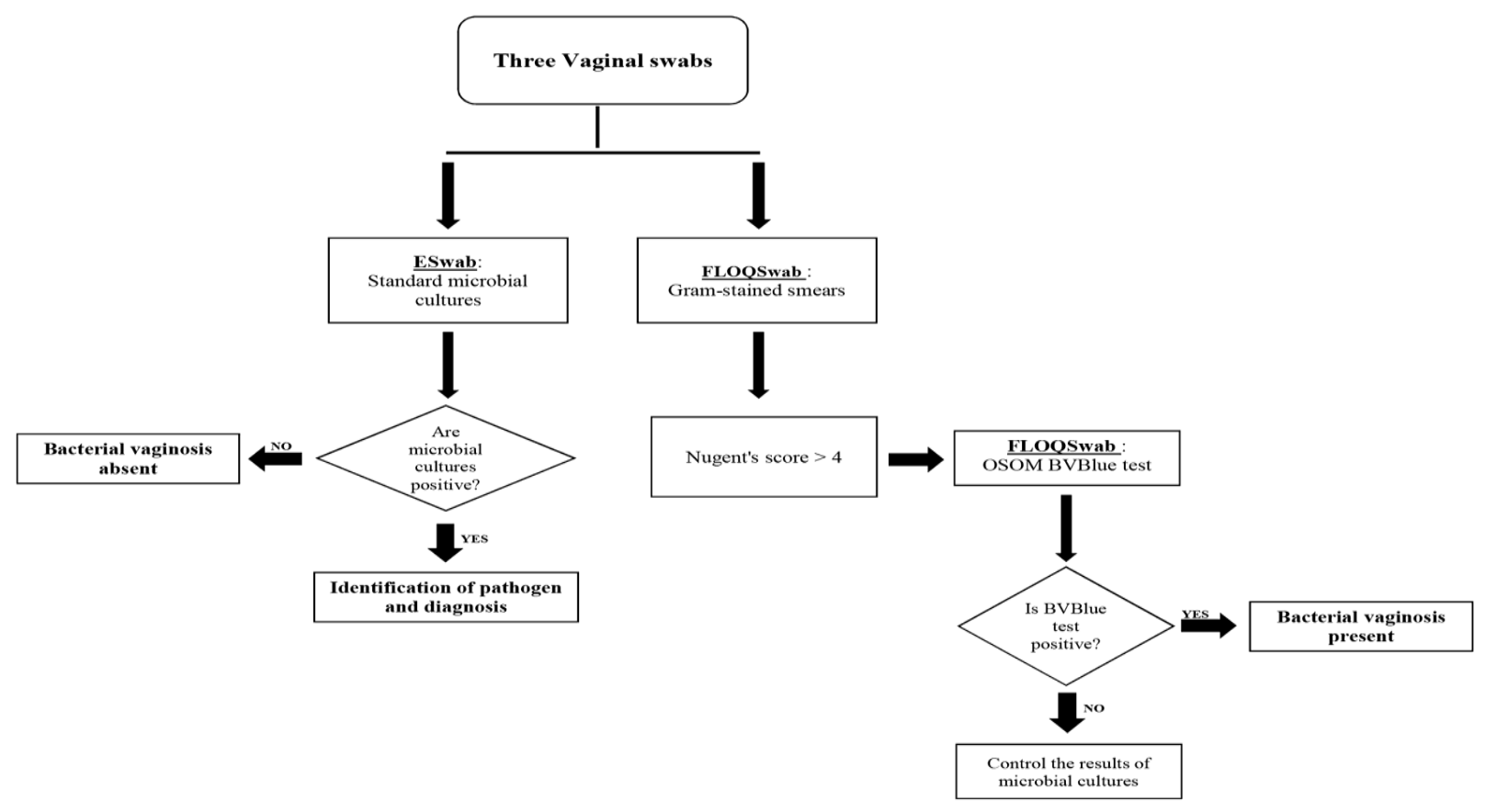


Collectively, we can speculate that BVBlue test does not appear to be a good screening test in our population. In fact, previous studies demonstrated that vaginal flora of women affected by BV differs by race/ethnicity, increasing the variability of the results (Royce et al., 1999; Jespers et al., 2012; Srinivasan et al., 2012). Our data suggest that the best approach to diagnose BV is, for Nugent's score higher than four, to perform the BVBlue test and standard cultures including selective media for G. vaginalis.

The execution of the BVBlue test in samples presenting an intermediate Nugent's score (between 4 and 6) could be useful to obtain indications regarding initial changes of vaginal flora.

In our study, G. vaginalis was isolated and identified in 35 samples with intermediate Nugent's score, suggesting a modification of vaginal microbiota with high probability to shift to BV but not necessarily the presence of infection.

G. vaginalis seems to play a key role in the formation of structured polymicrobial biofilm, which represents a hallmark of bacterial vaginosis (Kenyon and Osbak, 2014). Figure 1 depicts our proposed workflow showing the steps to improve BV diagnosis.

The presence of sialidase is not uniform, since its enzymatic activity was detected in only 75$84 \%$ of women with bacterial vaginosis (Briselden et al., 1992; Cauci et al., 1998; Marconi et al., 2012).

A potential source of BVBlue test falsenegatives could be represented by potential sialidase-negative $G$. vaginalis strains (Santiago et al., 2011; Janulaitiene et al., 2017), or by the presence of a small amount of anaerobic Gram-negative bacteria, such as Atopobium, Prevotella, Porphyromonas,
Mobilincus, and Bacteroides spp., which are known to produce sialidase. Recently, molecular diagnostic approaches have been used to study the vaginal biota in women affected by BV, showing that not a single but rather several species are present in the vaginal flora (Obata-Yasuoka et al., 2002; Ling et al., 2010; Srinivasan et al., 2012; Rumyantseva et al., 2015; Virtanen et al., 2017).

Alternative diagnostic techniques, such as gas-liquid chromatography and liquid preparation Papanicolaou smears, have been suggested as alternatives to standardized Gram stain methodology due to their practical advantage (Davis et al., 1997; Lamont et al., 1999; Wolrath et al., 2002; Barouti et al., 2013; Nenadić et al., 2015; Martínez-Girón et al., 2017).

However, they require significant changes in the approach of reading vaginal smears thus they might become highly valuable in the future.

Our study presents some limitations. First, this work was performed in a single hospital, and a larger number of specimens should be investigated to improve the accuracy of our results.

Second, molecular diagnostic tests, although expensive, could be useful to ascertain the discrepancy of the results between Gram staining and microbial cultures/BVBlue test performed for the same subject.

In conclusion, given the heterogeneity of bacterial vaginosis, BVBlue test, not alone but rather along with culture-based methods and scored Gram staining, can increase early diagnosis of bacterial vaginosis in our population. Future research should be conducted to improve the performance of BVBlue test. 


\section{Acknowledgments}

We gratefully acknowledge Laura Colombo, Marco Santambrogio, Elena Crippa, Antonio Pacifico, and Silvio Caimi from Desio Hospital for technical support.

We also thank Dr. Elena Intra for reviewing the manuscript.

\section{Compliance with ethical standards}

\section{Funding}

No specific funding was used for this research.

\section{Conflict of interest}

The authors declare that they have no conflict of interest.

\section{Ethical approval}

This article did not contain any studies with human participants and/or animals.

\section{Informed consent}

Written informed consent was obtained from all women recruited.

\section{References}

Bagnall, P., Rizzolo, D., 2017. Bacterial vaginosis: A practical review. JAAPA. doi: 10.1097/01.JAA.0000526770.60197.

Barouti, E., Farzaneh, F., Sene, A.A., Tajik, Z., Jafari, B., 2013. The pathogenic microorganisms in papanicolaou vaginal smears and correlation with inflammation. J. Family Reprod. Health. 7(1), 23-27.

Bhalla, P., Chawla, R., Garg, S., et al., 2007. Prevalence of bacterial vaginosis among women in Delhi, India. Indian J. Med. Res. 125(2), 167-172.
Bradshaw, C.S, Morton, A.N., Garland, S.M., et al., 2005. Evaluation of a point-of-care test, BVBlue, and clinical and laboratory criteria for diagnosis of bacterial vaginosis. J. Clin. Microbiol. 43(3), 13041308.

Briselden, A.M., Moncla, B.J., Stevens, C.E., Hillier, S.L., 1992. Sialidases (neuraminidases) in bacterial vaginosis and bacterial vaginosis-associated microflora. J. Clin. Microbiol. 30(3), 663666.

Cauci, S., Driussi, S., Monte, R., et al., 1998. Immunoglobulin A response against Gardnerella vaginalis hemolysin and sialidase activity in bacterial vaginosis. Am. J. Obstet. Gynecol. 178(3), 511-515.

Davis, J.D., Connor, E.E., Clark, P., Wilkinson, E.J., Duff, P., 1997. Correlation between cervical cytologic results and Gram stain as diagnostic tests for bacterial vaginosis. Am. J. Obstet. Gynecol. 177(3), 532-535.

Gaydos, C.A., Beqaj, S., Schwebke, J.R., et al., 2017. Clinical Validation of a Test for the Diagnosis of Vaginitis. Obstet. Gynecol. 130(1), 181-189.

Haggerty, C.L., Hillier, S.L., Bass, D.C., Ness, R.B., 2004. PID Evaluation and Clinical Health study investigators. Bacterial vaginosis and anaerobic bacteria are associated with endometritis. Clin. Infect. Dis. 39(7), 990-995.

Hillier, S.L., Krohn, M.A., Cassen, E., et al., 1995. The role of bacterial vaginosis and vaginal bacteria in amniotic fluid infection in women in preterm labor with intact fetal membranes. Clin. Infect. Dis. 20, S276-278.

Howe, L., Wiggins, R., Soothill, P.W., et al., 1999. Mucinase and sialidase activity of the vaginal microflora: implications for the pathogenesis of preterm labour. Int. J. STD AIDS. 10(7), 442-447.

Janulaitiene, M., Paliulyte, V., Grinceviciene, S., et al., 2017. Prevalence and distribution of Gardnerella vaginalis subgroups in women with and without bacterial vaginosis. BMC Infect Dis. 17(1), 394. 
Jespers, V., Menten, J., Smet, H., et al., 2012. Quantification of bacterial species of the vaginal microbiome in different groups of women, using nucleic acid amplification tests. BMC Microbiol. 12: 83.

Kampan, N.C., Suffian, S.S., Ithnin, N.S., et al., 2011. Evaluation of $\mathrm{BV}^{\circledR}$ Blue Test Kit for the diagnosis of bacterial vaginosis. Sex Reprod Healthc. 2(1), 1-5.

Kenyon, C.R., Osbak, K., 2014. Recent progress in understanding the epidemiology of bacterial vaginosis. Curr. Opin. Obstet. Gynecol. 26(6), 448-54.

Khatoon, R., Jahan, N., Ahmad, S., Rabbani, T., 2013. Comparison of OSOM BV Blue test with conventional methods for diagnosis of bacterial vaginosis. Af. J. Microb. Res. 7(28), 3698-3703.

Kusters, J.G., Reuland, E.A., Bouter, et al., 2015. A multiplex real-time PCR assay for routine diagnosis of bacterial vaginosis. Eur. J. Clin. Microbiol. Infect. Dis. 34(9): 1779-1785.

Lamont, R.F., Hudson, E.A., Hay, P.E., et al., 1999. A comparison of the use of Papanicolaou-stained cervical cytological smears with Gram-stained vaginal smears for the diagnosis of bacterial vaginosis in early pregnancy. Int. J. STD AIDS. 10(2), 93-97.

Ling, Z., Kong, J., Liu, F., et al., 2010. Molecular analysis of the diversity of vaginal microbiota associated with bacterial vaginosis. BMC Genomics. 11:488.

Lokken, E.M., Balkus, J.E., Kiarie, J., et al., 2017. Association of Recent Bacterial Vaginosis with Acquisition of Mycoplasma genitalium. Am. J. Epidemiol. 186(2), 194-201.

Madhivanan, P., Krupp, K., Li, T., et al., 2014. Performance of BVBlue rapid test in detecting bacterial vaginosis among women in Mysore, India. Infect. Dis. Obstet. Gynecol. doi: 10.1155/2014/908313.

Marconi, C., Donders, G.G., Parada, C.M., Giraldo, P.C., da Silva, M.G., 2013. Do Atopobium vaginae, Megasphaera spp. and Leptotrichia spp. change the local innate immune response and sialidase activity in bacterial vaginosis? Sex Transm. Infect. 89(2), 167-173.

Martínez-Girón, R., Martínez-Torre, C., van Woerden, H.C., Zapico-Ortíz, N., 2017. Observation of an association between ground-glass-like globules in Papanicolaou smears and bacterial vaginosis. Cytopathology. 28(3), 241242.

Moncla, B.J., Braham, P., Hillier, S.L., 1990. Sialidase (neuraminidase) activity among gram-negative anaerobic and capnophilic bacteria. J. Clin. Microbiol. 28(3), 422425.

Myziuk, L., Romanowski, B., Johnson, S.C., 2003. BVBlue test for diagnosis of bacterial vaginosis. J. Clin. Microbiol. 41(5), 1925-1928.

Nenadić, D.B., Pavlović, M.D., Motrenko, T., 2015. A novel microscopic method for analyzing Gram-stained vaginal smears in the diagnosis of disorders of vaginal microflora. Vojnosanit Pregl. 72(8), 670676.

Nugent, R.P., Krohn, M.A., Hillier, S.L., 1991. Reliability of diagnosing bacterial vaginosis is improved by a standardized method of gram stain interpretation. J. Clin. Microbiol. 29(2), 297-301.

Obata-Yasuoka, M., Ba-Thein, W., Hamada, H., Hayashi, H., 2002. A multiplex polymerase chain reaction-based diagnostic method for bacterial vaginosis. Obstet. Gynecol. 100(4), 759-764.

Puapermpoonsiri, S., Kato, N., Watanabe, K., et al., 1996. Vaginal microflora associated with bacterial vaginosis in Japanese and Thai pregnant women. Clin. Infect. Dis. 23(4), 748-752.

Royce, R.A., Jackson, T.P., Thorp, J.M., et al., 1999. Race/ethnicity, vaginal flora patterns, and $\mathrm{pH}$ during pregnancy. Sex Transm. Dis. 26(2), 96-102.

Rumyantseva, T.A., Bellen, G., Romanuk, T.N., et al., 2015. Utility of microscopic techniques and quantitative real-time polymerase chain reaction for the 
diagnosis of vaginal microflora alterations. Low Genit. Tract Dis. 19(2), 124-128.

Santiago, G.L., Deschaght, P., El Aila, N., et al., 2011. Gardnerella vaginalis comprises three distinct genotypes of which only two produce sialidase. Am. J. Obstet. Gynecol. 204(5), 450.

Shujatullah, F., Khan, H.M., Khatoon, R., Rabbani, T., Malik, A., 2010. An evaluation of OSOM BV blue test in the diagnosis of bacterial vaginosis. As. Pac. J. Tropic. Med. 3(7), 574-576.

Smayevsky, J., Canigia, L.F., Lanza, A., Bianchini, H., 2001. Vaginal microflora associated with bacterial vaginosis in nonpregnant women: reliability of sialidase detection. Infect Dis. Obstet. Gynecol. 9(1), 17-22.

Spiegel, C.A., 1991. Bacterial vaginosis. Clin. Microbiol. Rev. 4(4), 485-502.

Spiegel, C.A., Amsel, R., Holmes, K.K., 1983. Diagnosis of bacterial vaginosis by direct gram stain of vaginal fluid. J. Clin. Microbiol. 18(1), 170-177.

Srinivasan, S., Hoffman, N.G., Morgan, M.T., et al., 2012. Bacterial communities in women with bacterial vaginosis: highresolution phylogenetic analyses reveal relationships of microbiota to clinical criteria. PLoS One. 7(6):e37818.

Stephan, C., Wesseling, S., Schink, T., Jung, K., 2003. Comparison of eight computer programs for receiver-operating characteristic analysis. Clin. Chem. 49(3), 433-439.

Sweet, R.L., 1995. Role of bacterial vaginosis in pelvic inflammatory disease. Clin. Infect. Dis. 20, S271-275.

Virtanen, S., Kalliala, I., Nieminen, P., Salonen, A., 2017. Comparative analysis of vaginal microbiota sampling using 16S rRNA gene analysis. PLoS One. 12(7), e0181477.

Von Nicolai, H., Hammann, R, Salehnia, S, Zilliken, F., 1984. A newly discovered sialidase from Gardnerella vaginalis. Zentralbl. Bakteriol. Mikrobiol. Hyg. A. 258(1), 20-26.

Wang, J., 2000. Bacterial vaginosis. Prim. Care Update Ob. Gyns. 7(5), 181-185.

Wiggins, R., Crowley, T., Horner, P.J., et al., 2000. Use of 5-bromo-4-chloro-3-indolylalpha-D-N-acetylneuraminic acid in a novel spot test to identify sialidase activity in vaginal swabs from women with bacterial vaginosis. J. Clin. Microbiol. 38(8), 3096-3097.

Wiggins, R., Hicks, S.J., Soothill, P.W., et al., 2001. Mucinases and sialidases: their role in the pathogenesis of sexually transmitted infections in the female genital tract. Sex Transm. Infect. 77(6), 402-408.

Wolrath, H., Borén, H., Hallén, A., Forsum, U., 2002. Trimethylamine content in vaginal secretion and its relation to bacterial vaginosis. APMIS. 110(11), 819-824.

\section{How to cite this article:}

Jari Intra, Cecilia Sarto, Natalia Tiberti, Claudia Siracusa, Cinzia Savarino, Chiara Fania and Paolo Brambilla. 2018. Rapid Detection of Sialidase Activity for the Diagnosis of Bacterial Vaginosis. Int.J.Curr.Microbiol.App.Sci. 7(08): 3898-3908. doi: https://doi.org/10.20546/ijcmas.2018.708.401 